\title{
Case Files of the Medical Toxicology Fellowship at Banner Good Samaritan Medical Center in Phoenix, AZ: Methemoglobinemia Following Dapsone Exposure
}

\author{
Joshua Canning • Michael Levine
}

Published online: 13 April 2011

(C) American College of Medical Toxicology 2011

\begin{abstract}
Acquired methemoglobinemia is a common, potentially fatal syndrome that can occur as a result of exposure to numerous xenobiotics. A case report of a 14-month-old female who developed methemoglobinemia following a dapsone ingestion. The child was treated with numerous boluses of methylene blue and ultimately required a continuous infusion of methylene blue. The common causes of methemoglobinemia, as well as the underlying pathophysiology, diagnosis, and treatment strategies are discussed.
\end{abstract}

Keywords Methemoglobinemia $\cdot$ Methemoglobin .

Dapsone $\cdot$ Cyanosis $\cdot$ Poisoning $\cdot$ Methylene blue

\section{Case Presentation}

A 14-month-old female infant presented to the emergency department (ED) with a chief complaint of cyanosis. The child had no significant past medical history and appeared normal throughout the prior day, except for some mild rhinorrhea and a single episode of non-bloody, non-bilious emesis the night prior to admission. An additional episode of emesis occurred the morning of admission. There were no other complaints, and the remainder of the review of systems was negative. The patient's mother noted cyanosis around the face and lips, prompting evaluation in the ED. The patient's room air oxygen saturation by pulse oximetry $\left(\mathrm{SpO}_{2}\right)$ was $87 \%$, and a chest X-ray was negative. She was transferred to a pediatric tertiary care center for further evaluation of suspected congenital heart disease.

J. Canning $\cdot$ M. Levine $(\bowtie)$

Department of Medical Toxicology,

Banner Good Samaritan Medical Center,

Phoenix, AZ, USA

e-mail: Michael.Levine@bannerhealth.com
On arrival to the referral center, the child was noted to be tachycardic, tachypneic, and cyanotic in mild respiratory distress. The pertinent abnormal vital signs were a heart rate of 212 beats per minute and a respiratory rate of 48 breaths per minute. The $\mathrm{SpO}_{2}$ on $15 \mathrm{~L}$ of oxygen administered via a non-rebreather mask was $88 \%$. The remainder of the physical examination was unremarkable. The venous blood gas (VBG) drawn on arrival revealed a $\mathrm{pH}$ of 7.42, a $\mathrm{pCO}_{2}$ of $30 \mathrm{mmHg}$, a $\mathrm{pO}_{2}$ of $43 \mathrm{mmHg}, \mathrm{HCO}_{3}$ of $18.8 \mathrm{mmol} / \mathrm{L}$, and methemoglobin, "high." The initial venous blood drawn for the laboratory testing was noted to have a brownish hue. The hemoglobin concentration was $13.0 \mathrm{~g} / \mathrm{dL}$, and the remainder of the baseline complete blood count and comprehensive metabolic profile were normal.

Given the elevated methemoglobin fraction and clinical picture consistent with methemoglobinemia, the child received a $2-\mathrm{mg} / \mathrm{kg}$ IV bolus of methylene blue. The child's symptoms improved promptly and a repeat examination of the patient approximately $1 \mathrm{~h}$ later revealed an asymptomatic, playful child without cyanosis. A repeat VBG at this time revealed a methemoglobin fraction of $13.3 \%$. The child was transferred to the PICU for further observation.

What Is the Pathophysiologic Basis for Methemoglobin Formation?

Methemoglobin may occur from many potential causes. Regardless of the cause or predisposing condition, the final pathway is similar: oxidation of deoxyhemoglobin, converting the ferrous $\left(\mathrm{Fe}^{2+}\right)$ ion in heme to the ferric $\left(\mathrm{Fe}^{3+}\right)$ valence. This oxidized species of hemoglobin is unable to bind to oxygen and instead binds to a molecule of water [1, 2]. Normally, when oxygen binds to deoxyhemoglobin, an electron is shared between the iron in heme and the oxygen molecule, thus, the iron is reversibly oxidized. When $\mathrm{O}_{2}$ is 
released, the electron remains with the iron, returning it to the ferrous state. When oxygen dissociates from hemoglobin, it may sometimes remove the shared electron, leaving the iron in its oxidized ferric $\left(\mathrm{Fe}^{3+}\right)$ valence state. This process is referred to as auto-oxidation and is the same mechanism by which many xenobiotics can form methemoglobin. Since only reduced, ferrous $\left(\mathrm{Fe}^{2+}\right)$ iron is able to bind and carry oxygen, the oxidized methemoglobin $\left(\mathrm{Fe}^{3+}\right)$ must receive an electron from another source to restore its oxygen-carrying potential.

How Does the Body Regulate Methemoglobin Levels?

Red blood cells (RBCs) are more vulnerable to oxidative insult than other tissues in the body [3]. In RBCs, there are both major $(95 \%)$ and minor $(5 \%)$ pathways to reduce methemoglobin back to its oxygen-carrying state. Cytochrome- $b_{5}$ reductase (also known as NADH methemoglobin reductase) is the primary pathway involved in this reduction [1-5]. An additional enzyme, nicotinamide adenine dinucleotide phosphate (NADPH) methemoglobin reductase, is also capable of reducing methemoglobin; although its activity is quite limited during normal physiologic conditions, it becomes highly significant in the presence of methylene blue (see Fig. 1). While both of these enzymes ultimately reduce methemoglobin to deoxyhemoglobin, they obtain the electrons from different sources. Cytochrome- $b_{5}$ reductase utilizes NADH formed in glycolysis as an electron donor to "replace" ferric iron's missing electron. In contrast, NADPH methemoglobin reductase utilizes NADPH formed by the hexose monophosphate shunt by the enzyme glucose-6-phosphate dehydrogenase (G6PD) to reduce methylene blue to leukomethylene blue, which in turn donates an electron to reduce methemoglobin [6]. As discussed below, a deficiency in G6PD has important implications for the treatment of methemoglobinemia $[1,4]$.

As non-enzymatic antioxidants, ascorbic acid, reduced glutathione, flavin, cysteine, and tetrahydropterin can also play small roles in the reduction of methemoglobin [3]. Methemoglobin is always present to some degree in equilibrium with deoxyhemoglobin; the reduction of methemoglobin occurs at approximately $15 \%$ per hour [3]. For instance, assuming no further oxidant stress, an individual with a methemoglobin fraction of $20 \%$ would be expected to have a methemoglobin fraction of $17 \% 1 \mathrm{~h}$ later. The decline in methemoglobin concentrations follows first order kinetics [3].

Methemoglobinemia occurs when the formation of methemoglobin (via auto- or xenobiotic-induced oxidation of hemoglobin) occurs at a faster rate than cytochrome- $b_{5}$ reductase, and other non-enzymatic pathways can reduce it back to deoxyhemoglobin $[1,2,7]$. Auto-oxidation from $\mathrm{O}_{2}$ forms a baseline methemoglobin fraction of approximately $0.5 \%$ [4]. An abnormally elevated methemoglobin fraction is generally defined as greater than $1 \%[3,8,9]$. Some oxidizing agents may increase methemoglobin formation by up to 1,000 times [1]. While an absolute concentration of $5 \mathrm{~g} / \mathrm{dL}$ of deoxyhemoglobin must be present for cyanosis to occur, only $1.5 \mathrm{~g} / \mathrm{dL}$ of methemoglobin must be present to develop cyanosis (approximately equal to $10-15 \%$ methemoglobin in a patient with normal hemoglobin levels) $[1,8$, 10, 11]. Clinically, skin and mucous membrane cyanosis may also develop a brown hue as the methemoglobin fraction increases [8]. It is important to realize that patients with baseline functional anemia (e.g., sickle cell anemia or carboxyhemoglobinemia) will become symptomatic at lower methemoglobin fractions [1]. In addition to decreased arterial blood oxygen content, as methemoglobin fractions increase greater than $10 \%$, there is a left shift of the oxygen dissociation curve leading to an impaired unloading of oxygen at the tissue level [1, 2, 4, 7, 9].

Low concentrations of methemoglobin usually do not cause clinically significant symptoms and generally go unrecognized. Methemoglobin fractions must be interpreted in the context of hemoglobin concentration. For example, at methemoglobin fractions up to $15 \%$, a patient with normal
Fig. 1 Methemoglobin $(m e t H b)$ reduction pathways

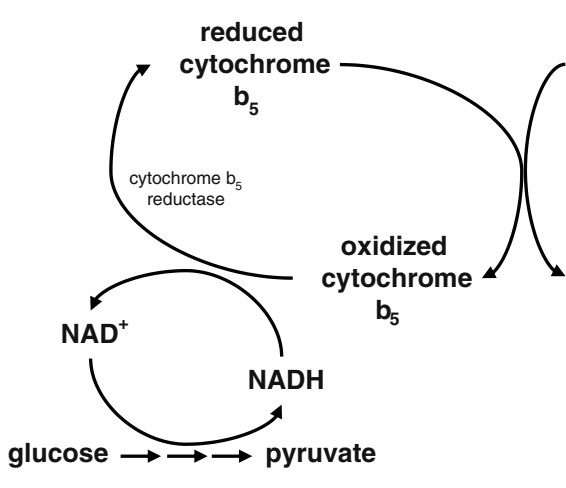

GLYCOLYSIS

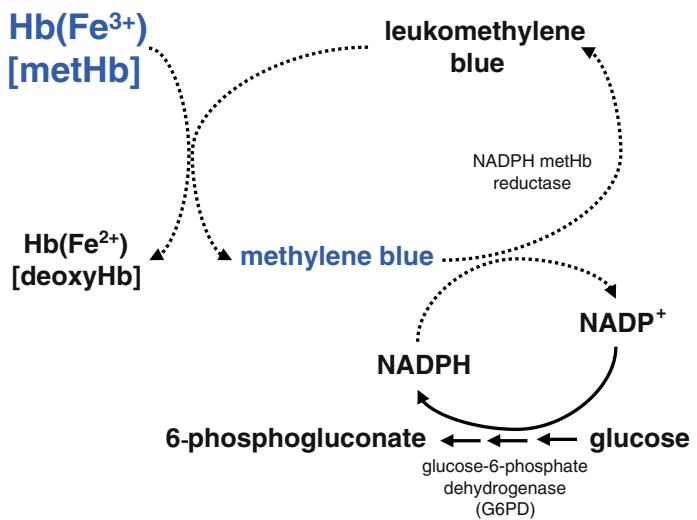

HEXOSE MONOPHOSPHATE SHUNT 
hemoglobin concentrations would be expected to have a few symptoms due to the presence of adequate functional hemoglobin, while an anemic patient with a similar fraction may be quite symptomatic [8]. Some patients have been reported to remain asymptomatic with methemoglobin fractions as high as $30-40 \%$ [7]. Morbidity and mortality at higher levels are generally a function of resultant tissue hypoxia. Headache, fatigue, and exercise intolerance can be expected with methemoglobin fractions $20-30 \%$, while fractions greater than $50 \%$ may cause seizures, cardiac dysrhythmias, and death [1]. Levels exceeding $70 \%$ are commonly lethal. One retrospective review found that most patients with methemoglobin greater than $8 \%$ were symptomatic, although most of the patients in the review had underlying anemia [1].

Besides anemia, the presence of other abnormal hemoglobins and comorbidities, such as underlying cardiac or pulmonary disease, sepsis, and acidosis, can significantly worsen the clinical picture for a given methemoglobin fraction $[1,11]$. Gastroenteritis and dehydration may also place younger children at risk for methemoglobin production [1].

What Are the Causes of Congenital Methemoglobinemia?

Certain conditions may predispose patients to methemoglobinemia due to a relative deficiency in their endogenous ability to reduce methemoglobin. In general, infants have up to $60 \%$ decreased activity of cytochrome- $b_{5}$ reductase compared to adults until about 4 months of age, making them predisposed to methemoglobinemia [3, 10, 12]. Additionally, neonates suffering from gastroenteritis may have increased nitrite formation in the gut $[3,12]$ and fetal hemoglobin is also more readily oxidized to methemoglobin [8]. Along with congenital heart disease, methemoglobinemia should be on the differential diagnosis of cyanosis in early infancy. Oxidizing xenobiotics should be avoided in a patient with known congenital predisposition to methemoglobinemia. Notably, while the presence of G6PD deficiency has implications in the treatment of methemoglobinemia (e.g., hemolysis with methylene blue exposure), G6PD deficiency itself does not cause methemoglobinemia [8].

Hemoglobin $\mathrm{M}$ disease $(\mathrm{HbM})$ is an autosomal dominant disorder characterized by a single amino acid substitution in $\alpha$ - or $\beta$-globin [3]. Heme is normally bound via histidine to the globin chain. When histidine is replaced with tyrosine as in $\mathrm{HbM}$, the heme moiety more readily auto-oxidizes creating the ferric $\left(\mathrm{Fe}^{3+}\right)$ state [12], which predisposes to methemoglobinemia in the presence of oxidizing substances [8]. Individuals who are heterozygous for $\mathrm{HbM}$ and normal hemoglobin will likely present with cyanosis at birth or within the first 6 months of life [10]. Homozygous states are thought to be incompatible with life [3, 8].

A deficiency in either enzymatic reducing pathway may also predispose to formation of methemoglobin. A homo- zygous deficiency in cytochrome- $b_{5}$ reductase may create a baseline of $10-50 \%$ methemoglobin fraction due to a reliance on the NADPH methemoglobin reductase and non-enzymatic pathways to reduce methemoglobin [8]. In fact, levels as high as $40 \%$ may be well tolerated, although the patient may remain with a persistent blue-brown cyanosis [8]. Heterozygous individuals may still only develop clinically significant methemoglobinemia under oxidative stress, albeit with a lower threshold [8]. There are several forms of cytochrome$b_{5}$ reductase deficiencies: type 1 is a less severe enzyme deficiency observed only in erythrocytes while individuals with type 2 appear to have a deficiency in all tissues $[10,13]$. A type 2 deficiency leads to a progressive neurologic deterioration and death in the first few years of life [10]. A patient may also be deficient in cytochrome- $b_{5}$ itself (type 4); all of these deficiencies appear to be inherited in an autosomal recessive pattern [3, 10]. Previously, a type 3 deficiency was also described; a subsequent investigation demonstrated that the type 3 deficiency was the same as a type 1. [14]

Given the relatively minor contribution of NADPH methemoglobin reductase in endogenous methemoglobin reduction, a deficiency in this enzyme by itself is not expected to cause a clinically significant methemoglobinemia. Notably though, these patients do not respond to methylene blue when used as a treatment for methemoglobinemia [3].

What Are the Causes of Xenobiotic-Induced Methemoglobinemia?

A number of stressors, particularly oxidant stress from various xenobiotics, can overwhelm the normally functioning reducing capabilities of cytochrome- $b_{5}$ reductase and other cellular systems [1]. This would be especially true in the setting of a deficient enzymatic reducing system as described above. Both chronic and acute illness as well as underlying comorbidities may also predispose the development of methemoglobinemia. While certain xenobiotics are capable of directly oxidizing ferrous $\left(\mathrm{Fe}^{2+}\right)$ iron to its ferric $\left(\mathrm{Fe}^{3+}\right)$ state, some of these act indirectly and must first be metabolized [2, 4]. Many cases of clinically significant methemoglobinemia involve the cumulative effect of various stressors, with or without a predisposing congenital condition. Comprehensive reviews of acquired etiologies of methemoglobinemia have been previously published $[1,3,8]$. Some of the more commonly encountered agents are presented below.

\section{Dapsone and Sulfamethoxazole}

Dapsone is a medication commonly used as a second-line agent behind sulfamethoxazole-trimethoprim (SMX-TMP) for prophylaxis and treatment of Pneumocystis jirovecii pneumonia in HIV patients, and is a relatively common cause of methemoglobinemia $[3,5,12]$. In one retrospec- 
tive review of acquired methemoglobinemia in an academic teaching hospital, dapsone was the most commonly identified cause of methemoglobinemia [1]. Dapsone is also used as a purported aphrodisiac [15] and as a treatment for leprosy [16]. Methemoglobinemia occurs indirectly via a metabolite formed by oxidative metabolism of dapsone in the cytochrome P450 system. This dapsone hydroxylamine metabolite is responsible for the actual oxidation of hemoglobin to methemoglobin $[5,15]$ The long half-life of this metabolite explains the prolonged duration of methemoglobinemia as well as the frequent need for repeated doses of methylene blue following dapsone exposure. Cimetidine's inhibition of the P450 isoenzymes responsible for dapsone metabolism (CYP3A4) explains its efficacy in the treatment of dapsone-induced methemoglobin [3]. In addition to methemoglobinemia, other adverse effects include doserelated hemolysis, peripheral neuropathy, and various idiosyncratic reactions, including hypersensitivity similar to SMX [12]. Both drugs have para-amino groups metabolized to a nitroso group via a hydroxylamine metabolite. While both dapsone and sulfonamides may cause methemoglobinemia, the hydroxylamine metabolite of SMX is significantly less potent than dapsone hydroxylamine for inducing methemoglobinemia in vitro $[1,5]$. The concomitant use of SMX-TMP may increase serum dapsone concentrations, thereby increasing the risk of methemoglobinemia [12].

\section{Local Anesthetics}

The local anesthetics benzocaine, prilocaine, lidocaine, and tetracaine indirectly oxidize hemoglobin to methemoglobin after they are first hydrolyzed to their corresponding amine metabolites $[2,3]$. Cetacaine ${ }^{\circledR}$ (benzocaine 14\%/ tetracaine $2 \%$ ), EMLA $^{\circledR}$ (lidocaine $2.5 \%$ /prilocaine $2.5 \%$ ), and Hurricaine ${ }^{\circledR}$ (benzocaine 20\%) are other commonly encountered preparations that have been reported to cause methemoglobinemia [1, 17, 18]. Topically, these agents rapidly enter the bloodstream through mucosal surfaces and are used for endotracheal intubation, transesophageal echocardiography, esophagogastroduodenoscopy, treatment of teething pain in infants, and in various topical anesthetic creams [6]. Benzocaine spray (20\%) is a common cause of serious methemoglobinemia with a mean peak methemoglobin fraction of $43.8 \%$ in one series $[1,6]$. Despite the manufacturer's recommendations of spraying the local anesthetic for less than $1 \mathrm{~s}$, this recommendation is not commonly heeded, which can result in methemoglobin formation following a single spray $[1,2]$. It is not currently possible to predict which patients are susceptible to the formation of methemoglobinemia from these agents [2]. Lidocaine-induced methemoglobinemia is relatively rare, although younger children exposed to large doses are thought to be most susceptible [2].
Nitrites/Nitrates, Nitroglycerin, and Nitric Oxide

Exposure to nitrites and nitrates can occur in a number of settings. Silver nitrate may be used for the treatment of burns while sodium nitrite is employed in the treatment of cyanide toxicity with the goal of inducing methemoglobinemia [1]. Amyl nitrate ("poppers") and isobutyl nitrite are potent vasodilators that may be used as drugs of abuse or as aphrodisiacs [1, 3, 15]. Most cases of nitrate-induced methemoglobinemia are reported in young infants being fed nitrate-contaminated well water. In the gut, bacteria convert dietary nitrates to nitrites, which in the setting of an immature methemoglobin reducing mechanism, predisposes this patient population [3, 8]. Despite prior reports describing methemoglobin fractions up to $9.6 \%$ associated with nitroglycerin infusion, the infusion rates used were up to three to ten times the normal rate $[7,19,20]$. A more recent series found no significant increase in methemoglobin fraction with infusion rates up to $2.12 \mu \mathrm{g} / \mathrm{kg} / \mathrm{min}$ [7]. Nitric oxide (NO) is used as an inhaled vasodilating agent that is selective for the pulmonary vasculature. In children with underlying congenital cardiac disease being treated with inhaled $\mathrm{NO}$, a statistically significant, dose-related rise in methemoglobin fractions was noted, but this did not appear to be clinically significant since the highest methemoglobin fraction measured was $3.9 \%$, and there was no significant increase in mortality [11].

\section{Other Xenobiotics}

Hydrazines are compounds that can cause methemoglobinemia as well as seizures, hepatic injury and hemolysis. They are commonly found in jet fuels as well as some pharmacologic agents and in monomethylhydrazine containing mushrooms such as Gyromitra esculenta (false morel). Aniline and its derivatives, including phenazopyridine, may also cause clinically significant methemoglobinemia $[1,3]$. Like dapsone, aniline may cause persistent methemoglobinemia despite methylene blue therapy [3]. Metoclopramide has been reported to cause methemoglobinemia in patients with renal failure [1,21]. Industrial exposure to arsine gas and naphthalene in mothballs both can cause hemolysis in addition to methemoglobinemia [3].

\section{What Diagnostic Studies Should Be Performed When Considering Methemoglobinemia?}

The consideration of methemoglobinemia on a differential diagnosis of cyanosis or hypoxia is imperative. In the setting of an unclear clinical picture, the appearance of a blood sample may be helpful, although it is not diagnostic. Blood from a patient with methemoglobinemia may appear to have a chocolate-brown color that does not change with 
exposure to air while normal venous blood would be expected to change to a bright red color when oxygen is bubbled through it $[4,7,8]$. The Kronenberg's "red-brown" test is a relatively simple, yet subjective, test involving placement of a drop of blood on a filter paper next to a drop of normal blood. Samples containing methemoglobin fractions $>10-15 \%$ are expected to appear brown compared to normal blood $[2,7,22]$.

\section{Arterial Blood Gas and Co-oximetry}

Co-oximetry evaluation should be ordered for any patient with suspected methemoglobinemia. Most commercially available laboratory co-oximeters shine at least four different wavelengths of light through a hemolyzed blood specimen to determine fractions of various hemoglobins, including methemoglobin, sulfhemoglobin, and carboxyhemoglobin. Abnormal hemoglobin species generally result in falsely elevated calculated oxygen saturation values on blood gas analysis [3]. Methemoglobin fractions may rise during storage at room temperature or after frozen storage and subsequent thawing of blood specimens prior to co-oximetric studies $[23,24]$. In one study, methemoglobin levels in frozen-thawed samples rose progressively from $1.8 \% 6 \mathrm{~h}$ after thawing, to nearly $11 \% 6$ days after thawing [23]. It is important to understand that values reported by co-oximetry and commonly referred to as "levels" are actually percentages of the total hemoglobin concentration, not absolute concentrations of methemoglobin in the blood, making them difficult to correlate with symptom severity. Also, many co-oximeters may detect sulfhemoglobin and report it as an elevated methemoglobin fraction [3]. Once a methemoglobin fraction is known, the determination of the actual blood concentration of methemoglobin (grams per deciliter) can be determined by multiplying the measured hemoglobin concentration.

\section{Conventional Pulse Oximetry}

Pulse oximeters function by shining a red light and a nearinfrared light through tissue, and subsequently measuring these lights at a detector. Because deoxyhemoglobin and oxyhemoglobin deflect light differently, the sensor is able to calculate the degree of deflection and calculate a saturation. Importantly, pulse oximetry does not measure methemoglobin. However, because methemoglobin does interfere with the deflection of these two beams, the pulse oximetry "misinterprets" this discrepancy as hypoxemia. A plateau exists, where despite increasing methemoglobin requirements, a saturation by pulse oximetry $\left(\mathrm{SpO}_{2}\right)$ of $\sim 85 \%$ is reported, regardless of the true oxygen status $[9,13]$.

When $\mathrm{SaO}_{2}$ values are known, a calculated oxygen saturation gap $\left(\mathrm{SaO}_{2}-\mathrm{SpO}_{2}\right)$ may be determined. In the setting of methemoglobinemia, one would expect an elevated gap due to falsely decreased $\mathrm{SpO}_{2}$ values [1, 2]. Importantly, methylene blue significantly alters pulse oximetry measurements of $\mathrm{SpO}_{2}$ following its administration [9]. Following methylene blue administration, the $\mathrm{SpO}_{2}$ may be as low as $65 \%$ and can persist artificially low for $1-2 \min$ [25].

\section{Pulse Co-oximetry}

Pulse co-oximetry is a newer technology that uses multiple LED wavelengths to attempt to noninvasively measure methemoglobin (and carboxyhemoglobin) fractions [9]. In a study examining the accuracy of a currently available pulse co-oximeter (Masimo Rainbow SET ${ }^{\circledR}$ Radical-7), as the true $\mathrm{SaO}_{2}$ decreased to $<95 \%$, the absolute methemoglobin fraction measurements may be overestimated by as much as $10-40 \%$ [9]. This inaccuracy may be improved on subsequent models of the machine [26]. Like conventional pulse oximetry, increased methemoglobin fractions lead to falsely decreased $\mathrm{SpO}_{2}$ measurements that underestimate the true $\mathrm{SaO}_{2}[1,9]$.

\section{(NADH) Cytochrome- $b_{5}$ Reductase Activity and Hemoglobin Electrophoresis}

In the setting of unexplained methemoglobinemia or lack of response to methylene blue, one should consider the diagnosis of an enzyme deficiency or a presence of an abnormal hemoglobin species. Specifically, cytochrome- $b_{5}$ reductase activity $[2,27]$ may be determined as well as hemoglobin electrophoresis examining specifically for $\mathrm{HbM}[2,10]$. Enzyme activity is determined by measuring the oxidation of NADH at a wavelength of $340 \mathrm{~nm}$ by spectrophotometry [10].

\section{Case Continuation}

Further discussion with the family revealed that the child lived with her parents and older siblings and there have been no sick contacts. The only medications in the home were acetaminophen, ibuprofen, and ampicillin. The mother denied giving the child any teething solutions or other home remedies and stated that the family receives drinking water from a municipal source.

A comprehensive urine drug and volatiles screen utilizing thin layer chromatography and gas chromatography/mass spectrometry (GC/MS) was positive only for acetone. Given the apparent lack of exposure to xenobiotics known to precipitate methemoglobinemia, a working diagnosis of methemoglobinemia precipitated by emesis in the setting of possible heterozygous NADH methemoglobin reductase deficiency was made. 
The following morning, a repeat methemoglobin fraction of $20.9 \%$ was obtained. Despite repeated boluses of methylene blue $(0.5-1 \mathrm{mg} / \mathrm{kg}$ IV) the child's methemoglobin fraction remained elevated with levels ranging from $11.1 \%$ to $19.1 \%$ over the next $24 \mathrm{~h}$. She received a total of five additional doses of methylene blue, and an initiation of a methylene blue continuous infusion was considered.

Given the refractory methemoglobinemia without a clear etiology, a second comprehensive urine drug screen was sent, and the results of the initial urine sample were reexamined. A selective ion search resulted in the detection of dapsone in both the initial and repeat urine samples.

The child was started on a continuous infusion of methylene blue at $1 \mathrm{mg} / \mathrm{h}$, with titration based on serial co-oximetry studies. Cimetidine was started and was scheduled every $6 \mathrm{~h}$. Over the subsequent $48 \mathrm{~h}$, the child's methemoglobin fraction remained $8.0-18.1 \%$.

\section{What Is GC/MS? How Does It Work?}

Gas chromatography/mass spectrometry involves the combination of two separate analytical techniques, and has become the criterion standard for drug detection. Gas chromatography permits the separation of a complex mixture with subsequent sequential presentation of the constituent parts to the analyzer, the mass spectrometer [28].

When used in comprehensive drug testing, the test sample, which is usually urine or blood, is inserted into an injection port on the gas chromatograph. The liquid is subsequently heated, thereby converting the unidentified chemicals to the gaseous form. The chemicals then move into a long chromatography column, where they are separated based on volatility. Those chemicals with lower volatility will separate out last, while those with higher volatility will separate out first.

As the chemicals leave the gas chromatograph, they enter the mass spectrometer for analysis. Upon entry into the mass spectrometer, the chemical is struck with electrons, causing the chemicals to become fragmented and ionized. A detector subsequently counts the number of ions at a particular mass, which ultimately results in the creation of a mass spectrum. Because each chemical produces a unique mass spectrum and chromatographic retention time, identification of each chemical is possible.

What Are Reasons Why a Particular Drug

May Not Be Detected on GC/MS and How Can Selective Ion Searching Help?

A drug may not be detectable on GC/MS for several reasons. First, if a drug or its metabolites are not water soluble or have no renal excretion, they will not be found in a urine sample. Second, the drug needs to be able to be extracted in an organic solvent to be detectable. Furthermore, some drugs are not volatilizable, while some others require a long duration of exposure to very high temperatures in order to be volatilized. Because of strain on the gas chromatograph's column, the technician frequently will only keep the column at very high temperatures (e.g., $300^{\circ}$ C) for a short span of time. Thus, if a drug has a particularly long retention time, it might not be detected with a routine analysis. Other drugs may decompose at high temperatures, which would limit the ability to detect the molecules. Furthermore, drugs of very small size are likely to not be detected, as most mass spectrometers are set to detect molecules above a certain molecular weight, to avoid interference with the helium used in the gas chromatograph. Lastly, certain characteristics of the gas chromatograph's column itself may not permit analysis of drugs with certain chemical characteristics (e.g., those with a large number of hydroxyl groups may not lend themselves to easy detection).

There is often some background interference when interpreting a GC/MS reading. Thus, if a drug is present, yet produces only a small spike on the mass spectrograph, it may be difficult to distinguish the spike from background interference. By looking for the unique pattern observed with a particular drug, selective ion searching may aid in identifying a suspected toxin when present in small concentrations. This technique may be useful if the clinician has a priori suspicion of a specific toxin.

What Are the Treatment Options for Methemoglobinemia?

Treatment of methemoglobinemia should be guided based on the severity of the condition and the underlying comorbid medical conditions. Methylene blue has traditionally been considered to be the primary antidote used to treat methemoglobinemia $[8,29]$. Depending on the clinical scenario, however, other treatment options may include cimetidine, exchange transfusion, $N$-acetylcysteine, and hyperbaric oxygen.

\section{Methylene Blue}

Methylene blue (tetramethylthionine chloride), is a thiazine dye that not only stimulates the hexose monophosphate shunt, but subsequently accepts electrons from NADPH and becomes reduced to leukomethylene blue via the enzyme NADPH methemoglobin reductase [8, 30, 31]. Leukomethylene blue, in turn, reduces methemoglobin to hemoglobin. The initial dose of methylene blue is $1-2 \mathrm{mg} / \mathrm{kg}$ of a $1 \%$ solution, administered intravenously over 5 min [8]. Additional doses of $1 \mathrm{mg} / \mathrm{kg}$ can be administered as needed for persistent methemoglobinemia, although the maximal total recommended dose is $7 \mathrm{mg} / \mathrm{kg}$. Refractory cases may warrant a continuous infusion [32]. 
There are several scenarios in which methylene blue may be ineffective in the treatment of methemoglobinemia. Because NADPH production is necessary in the formation of leukomethylene blue, patients who are deficient in glucose6-phosphate dehydrogenase (G6PD), the first enzyme in the hexose-monophosphate shunt, may not respond well to methylene blue. Furthermore, because of the potential to induce oxidant stress and subsequently exacerbate hemolysis in these patients, the use of methylene blue is considered at least relatively contraindicated in this patient population. Some have suggested that small doses $(<1 \mathrm{mg} / \mathrm{kg})$ can be cautiously attempted $[31,33]$. Similarly, patients who are deficient in NADPH methemoglobin reductase will not improve following therapy with methylene blue. Lastly, methylene blue will be ineffective if the underlying etiology is not methemoglobinemia, but rather sulfhemoglobinemia.

Methylene blue can be associated with several adverse effects, especially at higher doses. Chest pain, shortness of breath, tremor, and dysuria have all been described following methylene blue administration [8]. Furthermore, at doses exceeding $4 \mathrm{mg} / \mathrm{kg}$, methylene blue can itself serve as an oxidant stress, and thus produce further methemoglobinemia and hemolysis [8, 31]. Patients with G6PD deficiency may develop hemolysis at lower doses.

\section{Cimetidine}

Dapsone is metabolized via CYP3A4 to form several metabolites, including dapsone hydroxylamine. Cimetidine, an $\mathrm{H}_{2}$-receptor antagonist, inhibits CYP3A4, and should thus be considered for patients with dapsone-induced methemoglobinemia $[34,35]$.

\section{Miscellaneous Therapies}

For patients with life-threatening methemoglobinemia in whom methylene blue is contraindicated or ineffective, exchange transfusion may be required $[8,36]$. Hyperbaric oxygen can be used as a temporizing measure, but should not be viewed as a definitive therapy [37]. Due to its antioxidant effects, $\mathrm{N}$-acetylcysteine has been investigated in several studies as a treatment of methemoglobinemia. While efficacy was demonstrated in an in vitro model [37], such benefit was not observed in a volunteer study of subjects with nitrite-induced methemoglobinemia [38].

\section{Case Conclusion}

Over the next $24 \mathrm{~h}$, the methylene blue infusion was discontinued, and the child's methemoglobin fraction remained elevated at 5-6\%. Send out laboratory studies for nitrite/nitrate exposure and for unstable hemoglobin were negative. Methemoglobin reductase levels were sent, but unfortunately laboratory error prevented their completion. The patient's parents repeatedly denied any known means of possible exposure to dapsone. She remained asymptomatic and was subsequently discharged to home in the care of her parents. The source of dapsone was never discovered. The case was investigated for a potential child abuse, but no clear source was identified, and the child was released to the parents.

Conflict of Interest There are no financial litigations or other conflicts of interest involved in the preparation of this manuscript.

\section{References}

1. Ash-Bernal R, Wise R, Wright SM (2004) Acquired methemoglobinemia: a retrospective series of 138 cases at 2 teaching hospitals. Medicine 83:265-273

2. Guay J (2009) Methemoglobinemia related to local anesthetics: a summary of 242 episodes. Anesth Analg 108:837-845

3. Wright RO, Lewander WJ, Woolf AD (1999) Methemoglobinemia: etiology, pharmacology, and clinical management. Ann Emerg Med 34:646-656

4. Saha SA, Kordouni MR, Siddiqui M, Arora RR (2006) Methemoglobinemia-induced cardio-respiratory failure secondary to topical anesthesia. Am J Ther 13:545-549

5. Reilly TP, Woster PM, Svensson CK (1999) Methemoglobin formation by hydroxylamine metabolites of sulfamethoxazole and dapsone: implications for differences in adverse drug reactions. J Pharm Exp Ther 288:951-959

6. So TY, Farrington E (2008) Topical benzocaine-induced methemoglobinemia in the pediatric population. J Pediatr Health Care 22:335-339

7. Saxon SA, Silverman ME (1985) Effects of continuous infusion of intravenous nitroglycerine on methemoglobin levels. Am J Cardiol 56:461-464

8. Curry SC (1982) Methemoglobinemia. Ann Emerg Med 11:214-221

9. Feiner JR, Bickler PE, Mannheimer PD (2010) Accuracy of methemoglobinemia detection by pulse co-oximetry during hypoxia. Anesth Analg 111:143-148

10. Da-Silva SS, Sajan IS, Underwood JP (2003) Congenital methemoglobinemia: a rare cause of cyanosis in the newborn - a case report. Pediatrics 112:e158-e161

11. Hermon MM, Burda G et al (2003) Methemoglobin formation in children with congenital heart disease treated with inhaled nitric oxide after cardiac surgery. Intensive Care Med 29:447-452

12. Schiff DE, Roberts WD, Sue YJ (2006) Methemoglobinemia associated with dapsone therapy in a child with pneumonia and chronic immune thrombocytopenic purpura. J Pediatr Hematol Oncol 28:395-398

13. Turner MD, Karlis V, Glickman RS (2007) The recognition, physiology, and treatment of medication-induced methemoglobinemia: a case report. Anesth Prog 54:115-117

14. Nagai T, Shirabe K, Yubisui T et al (1993) Analysis of mutant NADH-cytochrome b5 reductase: apparent type III can be explained as type I with an unstable reductase. Blood 81:808-814

15. Lee SW, Lee JY, Lee KJ, Kim M, Kim MJ (1999) A case of methemoglobinemia after ingestion of an aphrodisiac, later proven as dapsone. Yonei Med J 40:388-391

16. Zosel A, Rychter K, Leikin JB (2007) Dapsone-induced methemoglobinemia: case report and literature review. Am $\mathrm{J}$ Ther 14:585-587 
17. Cetylite Industries, Inc. (2009) Cetacaine topical anesthetic spray. http://www.cetylite.com/cetacaine spray.html. Accessed 21 Dec 2010

18. Astrazeneca LP (2005) EMLA package insert. http://www1. astrazeneca-us.com/pi/EMLA.pdf. Accessed 21 Dec 2010

19. Gibson GR, Hunter JB, Raabe DS Jr et al (1982) Methemoglobinemia produced by high-dose intravenous nitroglycerin. Ann Intern Med 96:615-616

20. Husum B, Lindeburg T, Jacobsen E (1982) Methemoglobin formation after nitroglycerin infusion. Br J Anaesth 54:571

21. Karadsheh NS, Shaker Q, Ratroat B (2001) Metoclopramideinduced methemoglobinemia in a patient with co-existing deficiency of glucose-6-phosphate dehydrogenase and NADHcytochrome b5 reductase: failure of methylene blue treatment. Haematologica 86:659-660

22. Harley JD, Celermajer JM (1970) Neonatal methaemoglobinaemia and the "red-brown" screening-test. Lancet 296:1223-1225

23. Wallace KL, Curry SC (2002) Postcollection rise in methemoglobin level in frozen blood specimens. Clin Toxicol 40:91-94

24. Fairbanks VF (1980) A pitfall in hemoglobin electrophoresis. Artefactual minor unstable hemoglobin results from improper specimen handling. Am J Clin Pathol 73:245-247

25. Scheller MS, Unger RJ, Kelner MJ (1986) Effects of intravenously administered dyes on pulse oximetry readings. Anesthesiology 65:550-552

26. Feiner JR, Bickler PE (2010) Improved accuracy of methemoglobin detection by pulse co-oxemetry during hypoxia. Anesth Analg 111:1160-1167

27. Maran J, Guan Y, Ou CN, Prchal JT (2005) Heterogeneity of the molecular biology of methemoglobinemia: a study of eight consecutive patients. Haematologica 90:687-689

28. Niwa T (1995) Clinical mass spectrometry. Introduction Clinica Chimica Acta 241:3-12
29. do Nascimento TS, Pereira RO, de Mello HL et al (2008) Methemoglobinemia: from diagnosis to treatment. Rev Bras Anestesiol 58:651-654

30. Metz EN, Balcerzak SP, Sagone AL Jr (1976) Mechanism of methylene blue stimulation of the hexose monophosphate shunt in erythrocytes. J Clin Invest 58:797-802

31. Clifton J 2nd, Leikin JB (2003) Methylene blue. Am J Ther 10:289-291

32. Berlin G, Brodin B, Hilden JO et al (1984-1985) Acute dapsone intoxication: a case treated with continuous infusion of methylene blue, forced diuresis and plasma exchange. J Toxicol Clin Toxicol 22:537-548

33. Schuurman M, van Waardenburg D, DaCosta J et al (2009) Severe hemolysis and methemoglobinemia following fava beans ingestion in glucose-6-phosphate dehydrogenase deficiency: case report and literature review. Eur J Pediatr 168:779-782

34. Coleman MD, Coleman NA (1996) Drug-induced methaemoglobinaemia. Treatment issues. Drug Saf 14:394-405

35. Rhodes LE, Tingle MD, Park BK et al (1995) Cimetidine improves the therapeutic/toxic ratio of dapsone in patients on chronic dapsone therapy. Br J Dermatol 132:257-262

36. Jansen T, Barnung S, Mortensen CR et al (2003) Isobutyl-nitriteinduced methemoglobinemia; treatment with an exchange blood transfusion during hyperbaric oxygenation. Acta Anaesthesiol Scand 47:1300-1301

37. Wright RO, Magnami B, Shannon MW, Woolf AD (1996) Nacetylcysteine reduces methemoglobin in vitro. Ann Emerg Med 28:499-503

38. Tanen DA, LoVecchio F, Curry SC (2000) Failure of intravenous $\mathrm{N}$-acetylcysteine to reduce methemoglobin produced by sodium nitrite in human volunteers: a randomized controlled trial. Ann Emerg Med 35:269-273 